\title{
MANAGEMENT OF TEMPOROMANDIBULAR JOINT ARTHRITIS USING LOW LEVEL LASER THERAPY METHOD
}

\author{
Raafat Hassan Anwar Riad*
}

\begin{abstract} reduction in acute and chronic musculoskeletal pain. symptoms of temporomandibular joint (TMJ) arthritis. according to following groups:

(T1) before any treatment

(T2) after six sessions of laser therapy application.

(T3) after 12 laser therapy sessions applications.

(T4) one month after the last session application.
\end{abstract}

Background: Many studies have recommend using low-level laser therapy (LLLT) in pain

Objective: To clinically evaluate the effectiveness and validity of (LLLT) for management

Methodology: A clinical study included patients with TMJ arthritis signs and symptoms, were randomly categorized into two groups of ten patients each. Patients of the first group were subjected to pre adjusted (LLLT) over four weeks together with NSAIDs. Patients were evaluated clinically

The second group received only (NSAIDs). They were evaluated at the same time intervals of the first group.

Evaluation criteria were:

- Maximum mouth opening which measured in mm using ruler.

- Presence or absence of pain at TMJ area. The VAS method was used to quantify pain at TMJ area with palpation.

Results: Evaluation of maximum mouth opening in $\mathrm{mm}$ showed highly marked significant clinical improvement In (LLLT and NSAIDs) group. The same clinical improvement was noted in (NSAIDs) group. Comparison of results between the two groups showed a marked significant clinical improvement in pain reduction in favor of the (LLLT and NSAIDs) group during all evaluation periods. Same clinical improvement in pain reduction in favor for (LLLT and NSAIDs) group was noted in (VAS).

Conclusion. (LLLT) in patients with TMJ arthritis is safe and effective technique to be used.

KEYWORDS: Temporomandibular joint, TMJ, arthritis, LLLT.

\footnotetext{
* Assistant Professor of Oral and Maxillofacial Surgery, Armed Forces Medical Academy.
} 


\section{INTRODUCTION}

Temporomandibular disorders is a collective term to describe clinical signs and symptoms that involving muscles of mastication, temporomandibular joint and other associated structures ${ }^{[1]}$. Some studies show that $3-7 \%$ of the adult population seek medical care to decrease TMJ pain and dysfunction ${ }^{[2]}$.

Symptoms of (TMDS) range between $16 \%$ and $59 \%$ while the range of clinical signs lies between $33 \%$ and $86 \%$. Among patients with TMJ disorders only $11 \%$ have symptoms of TMJ arthritis ${ }^{[3,4]}$.

Super pulsed low-level laser therapy method (LLLT) may serve a good option as a non-invasive method in management of TMJ pain with a low cost for the patient.

Many authors have reported marked pain reduction with using low-level laser therapy teqnique in acute and chronic musculoskeletal associated pain conditions ${ }^{[5,6]}$. The results of many studies show no clinical improvements for any of following signs and symptoms which may be associated with TMDS such as: localized pre auricular swelling, muscle crumples and functional disorders.

The main limitation of the systematic metaanalyses study of (LLLT) method is the variation of clinical application, different emitting dosages, different beam wavelengths and different types of (LLLT) ${ }^{[5]}$.

The aim of this study is to clinically evaluate the validity and effectiveness of (LLLT) method in management of TMJ arthritis symptoms.

\section{METHODOLOGY}

This was a clinical study that included twenty patients with signs and symptoms of TMJ arthritis. Patients were collected from the outpatient clinics of the referral military hospital in Cairo (Egypt) over one year.

\section{Inclusion criteria of patients were}

1) Patients with TMJ arthritis, diagnosed according to history of signs and symptoms and physical examination of preauricular area, together with other diagnostic imaging utilities ${ }^{[7]}$.

2) The presence of signs and symptoms like pain or clicking at the TMJ area, or functional limitations.

3) Patients should have no history of previous surgery involving the TMJ area.

\section{Exclusion criteria of patients were}

1) Patients with past history of maxillofacial trauma.

2) Presence of other soft or hard tissues maxillofacial pathological lesions.

3) Presence of systemic rheumatic diseases like rheumatoid arthritis.

\section{Patients were randomly categorized into two groups of ten each;}

- Patients of the first group were treated with pre adjusted LLLT sessions over four weeks' time lasting period, together with standard NSAIDs. Therapy received three times a week and lasting four weeks, totaling twelve sessions.

Diode Laser or (Ga As) with $100 \mathrm{~mW}$ device was adjusted to emit a pulsed infrared beam of $810 \mathrm{~nm}$ wavelength according to previous studies (Mustang 2000z, Moscow, Russia) [Figure I].

The laser was adjusted and applied in contact mode with a peak power of $80 \mathrm{~W}, 50 \mathrm{~mW}$ and the average power at repetition rate of $1500 \mathrm{~Hz}$, the pulse time length was $1 \mathrm{MS}$, and energy was $6 \mathrm{~J}$ per point, $3.4 \mathrm{~J} / \mathrm{cm}^{2}$, and application spot size equals $1.76 \mathrm{~cm}^{2}$, for 2 minutes per point.

Three points of beam application around the TMJs areas were posterior, anterior, and superior to the mandibular condylar area [Figure II]. 


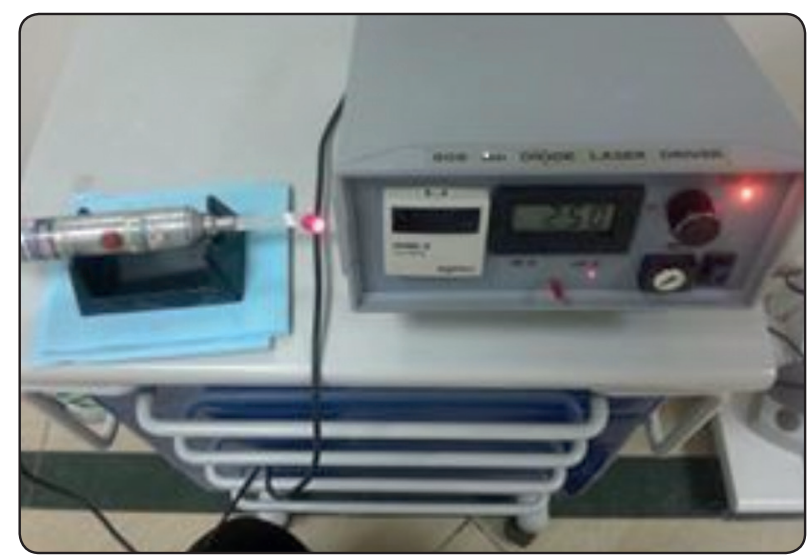

Fig. (I) Showing Diode Laser (Ga As) $100 \mathrm{~mW}$ ) device.

Patients were evaluated clinically according to following groups:

- (T1) clinical evaluation before treatment according to following groups.

- (T2) clinical evaluation after six sessions of laser application therapy.

- (T3) clinical evaluation after 12 laser application sessions.

- (T4) clinical evaluation one month after the last application
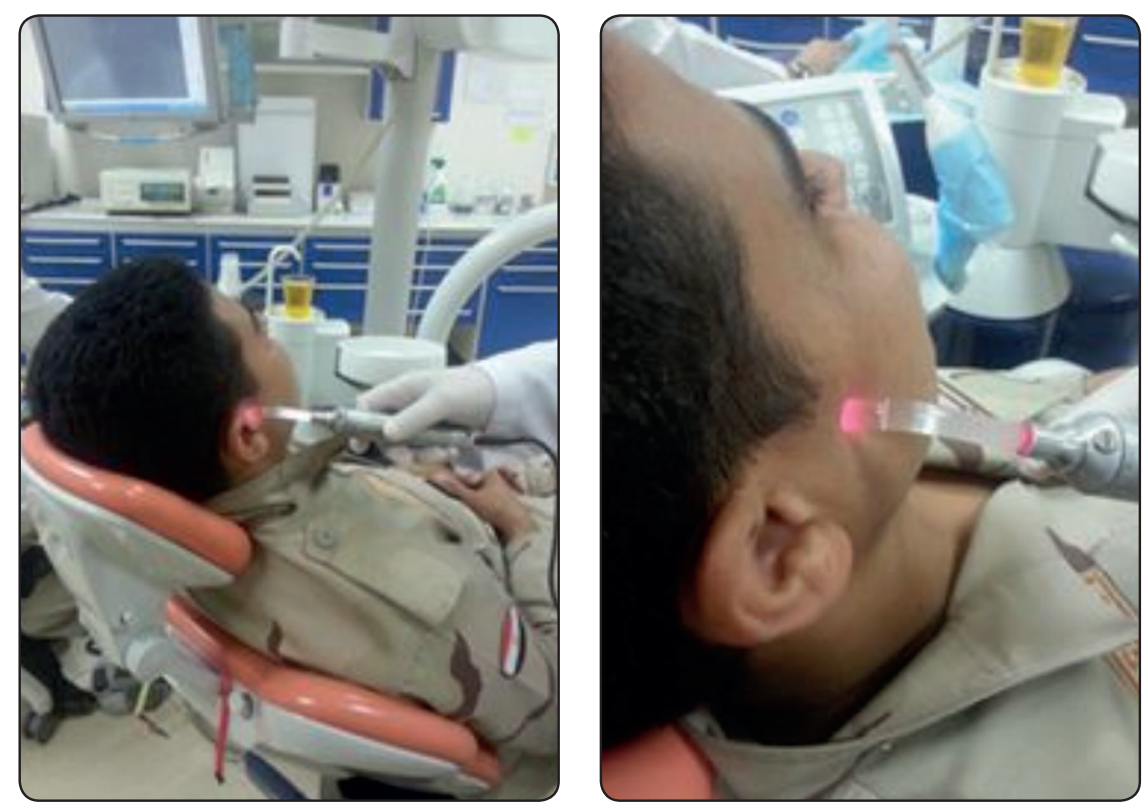

\section{Patients of the second group treated only with} NSAIDs for pain treatment of TMJ arthritis. They were evaluated at the same time intervals of the first group.

At clinical evaluation session, the maximum mouth opening was evaluated and measured in millimeters using ruler and compared to normal range and to other groups.

Patients were asked to express the presence or absence of pain around auricular area during rest and during function before and after laser application.

The VAS method was the method of choice used to quantify, estimate and evaluate pain at auricular area with palpation.

Patients were asked to mark the peak of pain intensity on a $10-\mathrm{cm}$ horizontal pain scale, while the left side of the scale indicated no pain (zero), and the right side of the scale (10) indicated the maximum possible pain.

Each patient in the study was informed about the procedures and informed about the study and signed an informed consent form before the commencement of the study.
Fig. (II) Showing Application of probe of Diode Laser at three point application at auricular area (anterior, superior and posterior). 


\section{Data analysis}

Data screen was done using Microsoft Excel program. Data were checked, coded and entered to the computer. Another data checking was done. Descriptive data analysis was done: mean and the standard deviation for data were calculated. Comparisons of the evaluation data between two groups were subjected to non-parametric (Friedman test and Mann-Whitney U test) statistical analysis method. Analysis of the data of results was done according to SPSS method (Statistical Package for Social Science version 15.0).

\section{RESULTS}

All chosen patients were males, and aged between 41 and 70 years old. The calculated mean age of the participants was 55.9 with (SD 9.3) years.

The mean age of the first group (LLLT and NSAIDs) was 56 with (SD 10) years, meanwhile the mean age of the second group (NSAIDs) was 55.8 with (SD 7.5) years. No statistical marked significant difference was found between both groups' data results regarding the age ( $\mathrm{p}$ value 0.82 ).

In the first group: evaluation of maximum mouth opening in $\mathrm{mm}$ showed a very highly marked significant clinical improvement during rest and function with ( $\mathrm{p}$ value $=0.000$ ). The same significant clinical improvement was noted in NSAIDs group ( $\mathrm{p}$ value 0.000 )

Comparison of evaluation results of (LLLT and NSAIDs) group and (NSAIDs) group showed a marked significant clinical improvement in favor of the (LLLT and NSAIDs) group during the three evaluation periods [table 1].

The (LLLT and NSAIDs) group showed a very highly significant clinical improvement in (VAS) Pain scale with ( $\mathrm{p}$ value 0.000 ) during the three clinical evaluation results. The same significant clinical improvement in results were noted in (NSAIDs) group with ( $\mathrm{p}$ value 0.000 ). Comparison of results between (LLLT and NSAIDs) group and (NSAIDs) group showed a significant clinical improvement in favor of the (LLLT and NSAIDs) group during the three clinical evaluation periods [table 2].

TABLE (1) Comparison of values of maximum mouth openings in mm in TMJ arthritis patients treated between (LLLT and NSAIDs) or only (NSAIDs).

\begin{tabular}{|l|c|c|c|}
\hline \multicolumn{1}{|c|}{ Variable / Group } & LLLT and NSAIDs Mean (SD) & NSAIDs (Mean (SD) & P value \\
\hline Maximum mouth opening in mm before treatment & $10.2(1.13)$ & $10(1.16)$ & 0.64 \\
\hline Maximum mouth opening in mm. First follow up & $30.8(2.5)$ & $22.6(3.13)$ & 0.000 \\
\hline Maximum mouth opening in mm. Second follow up & $34.6(3)$ & $27.6(2.6)$ & 0.000 \\
\hline Maximum mouth opening in mm. Third follow up & $38.4(2.3)$ & $30.6(3.13)$ & 0.000 \\
\hline
\end{tabular}

TABLE (2) Comparison between (VAS) Pain scales results in TMJ arthritis patients treated with (LLLT and NSAIDs) or only NSAIDs.

\begin{tabular}{|l|c|c|c|}
\hline \multicolumn{1}{|c|}{ Variable / Group } & LLLT and NSAIDs Mean (SD) & NSAIDs (Mean (SD) & P value \\
\hline VAS Pain scale before treatment & $8(0.82)$ & $8.2(0.92)$ & 0.69 \\
\hline VAS Pain scale after treatment. First evaluation period & $4.5(0.53)$ & $6.4(0.97)$ & 0.000 \\
\hline VAS Pain scale after treatment. Second evaluation period & $3.4(0.52)$ & $5.8(0.79)$ & 0.000 \\
\hline VAS Pain scale after treatment. Third evaluation period & $1.6(0.7)$ & $3.1(0.57)$ & 0.001 \\
\hline
\end{tabular}




\section{DISCUSSION}

This descriptive clinical study evaluate the effectiveness and validity of using (LLLT) to treat of TMJ arthritis symptoms.

Before reaching conclusions and recommendation based on the present study results, it is necessary to consider a number of potential limitations of this trial. Although the methodology of the device can be applied in different settings, these results were specific to patients attending to our referral military hospital and could not be considered generalizable. However, study findings were consistent and coherent, strongly indicating the external validity of the study.

In conclusion, using (LLLT) method in patients with symptomatic TMJ arthritis is safe and effective technique in relieving symptoms.

\section{REFERENCES}

1. de Leeuw R. Internal derangements of the temporomandibular joint. Oral Maxillofac Surg Clin North Am 2008;20(2):159-68.

2. Carlsson GE. Epidemiology and treatment need for temporomandibular disorders. J Orofac Pain 1999;13(4):232-7.

3. Mejersjö C, Hollender L. TMJ pain and dysfunction: relation between clinical and radiographic findings in the short and long-term. Scand J Dent Res 1984;92(3):241-8.

4. Tanaka E, Detamore MS, Mercuri LG. Degenerative disorders of the temporomandibular joint: etiology, diagnosis, and treatment. J Dent Res 2008;87(4):296-307.

5. Bjordal JM, Couppé C, Chow RT, Tunér J, Ljunggren EA. A systematic review of low level laser therapy with location-specific doses for pain from chronic joint disorders. Aust J Physiother 2003;49(2):107-16.

6. Ninomiya T, Hosoya A, Nakamura H, Sano K, Nishisaka T, Ozawa H. Increase of bone volume by a nanosecond pulsed laser irradiation is caused by a decreased osteoclast number and an activated osteoblasts. Bone 2007;40(1):140-8.

7. Gauer RL, Semidey MJ. Diagnosis and treatment of temporomandibular disorders. Am Fam Physician 2015; 91(6):378-86. 